\section{Commentary: Heat shock protein 90 inhibition and donor heart protection-a paradoxical concept?}

\author{
David J. Chambers, PhD
}

This study ${ }^{1}$ is of significant interest to those involved in cardiac transplantation, as it examines techniques to improve the viability (and therefore the potential availability) of donor hearts after circulatory death (DCD); specifically, the effect of a heat shock protein 90 inhibitor (HSP90i). For the average surgeon (as well as myself), it is likely that their understanding of the complexities of HSPs, and their action, are limited! A review by Soti and colleagues ${ }^{2}$ indicates that HSPs are chaperone molecules that protect other proteins subjected to cellular stress. This occurs by a variety of mechanisms, such as preventing denaturation or enhancing refolding of damaged proteins. Exposure to cellular stress (such as ischemia-reperfusion injury in the case of donor hearts used for cardiac transplantation) leads to increased induction/synthesis of HSPs (and particularly HSP90) by the activation of heat shock factor (HSF)-1. In the resting cell, HSP90 and HSF-1 are bound and thereby inactive. Stress leads to dissociation of this complex, leading to nuclear translocation of HSF-1 and subsequent HSP90 production, although these mechanisms are not completely understood. This paper assumes that the reader will have some basic understanding of the mechanism by which HSPs act, since the authors give no background into the role and mechanism of action of these molecules. An explanation of the paradoxical concept of improved protection by HSP90 inhibition (presumably by reduced

\footnotetext{
From Cardiac Surgery/Cardiac Surgical Research, The Rayne Institute (King's College London), Guy's and St Thomas' NHS Foundation Trust, St Thomas' Hospital, London, United Kingdom.

Disclosures: The author reported no conflicts of interest.

The Journal policy requires editors and reviewers to disclose conflicts of interest and to decline handling or reviewing manuscripts for which they may have a conflict of interest. The editors and reviewers of this article have no conflicts of interest.

Received for publication March 31, 2020; accepted for publication March 31, 2020.

Address for reprints: David J. Chambers, PhD, Cardiac Surgery/Cardiac Surgical Research, The Rayne Institute (King's College London), Guy's and St Thomas' NHS Foundation Trust, St Thomas' Hospital, 4th Floor, Lambeth Wing, London SE1 7EH, United Kingdom (E-mail: david.chambers@kcl.ac.uk).

J Thorac Cardiovasc Surg 2022;163:e201-2 0022-5223/\$36.00

Crown Copyright $(\subset) 2020$ Published by Elsevier Inc. on behalf of The American Association for Thoracic Surgery

https://doi.org/10.1016/j.jtcvs.2020.03.150
}

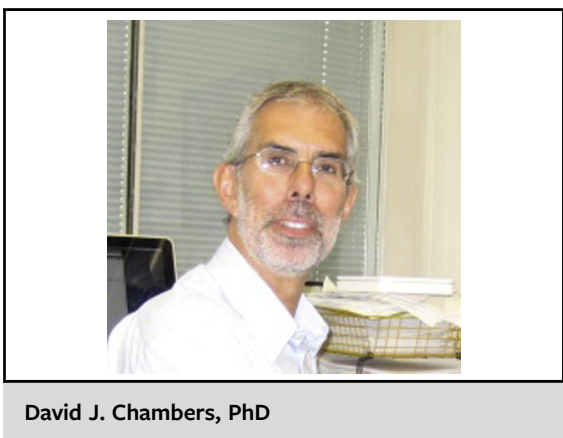

\section{CENTRAL MESSAGE \\ Heat shock protein 90 inhibition (paradoxically?) enhances cardi- oprotection in an experimental model of donor heart after cir- culatory death.}

expression/activation of HSP90) in contrast to the conferred cardioprotective role induced by the imposed ischemic stress leading to activation/synthesis of HSPs would benefit understanding and the manuscript enhanced if these basic physiological details could have been explained. The study would also have benefitted from some further experiments aimed at demonstrating what happens to HSP90 expression/activity during the course of the experimental protocol. Interestingly, in an earlier study by the authors, ${ }^{3}$ they were able to show increased expression of HSP70 as a result of using this inhibitor (but this was not examined in this study).

The study uses a Langendorff-perfused rat heart model of DCD and specifically incorporates the period of warm ischemic arrest that occurs in the clinical situation of heart transplantation before cardioplegic protection. Postischemia and arrest, functional assessment of the hearts indicates improved protection in HSP90i-treated hearts. One wonders why the authors chose this Langendorff preparation in preference to a "working" heart preparation. In the Limitations section of the article, it is stated that a study by Kearns and colleagues ${ }^{4}$ found that not all the DCD hearts showed a sustainable recovery! However, for clinical relevance, this is exactly the type of preparation that is required; if the transplanted heart (taken from a healthy donor animal as in this study) cannot generate sufficient cardiac output to overcome the systemic afterload, it will never be able to sustain the recipient patient!

Another potential limitation relates to the dose $(0.01 \mu \mathrm{mol} / \mathrm{L})$ of the HSP90i that was used (derived from Aceros and colleagues ${ }^{3}$ in cardiomyocytes); although this shows considerable benefit, it would be of interest to 
examine other doses in this organ preparation to determine potential optimum doses.

Overall, this is a study of considerable interest. However, it poses many more questions than it answers. There are a number of next steps to be taken; using a "working" heart preparation, perfusing these hearts with blood rather than a crystalloid perfusate, and examining the effects of the inhibitor in a more clinically relevant large animal preparation (perhaps using pigs subjected to the cardiopulmonary bypass procedures used during heart transplantation). This last point has recently been criticized in a Commentary of a recent publication of ours that questions the relevance of such large animal studies, with a central message that "animal research rarely benefits humans." However, my personal opinion is that such a step is vital to the process of translational medicine when incorporating drugs, like this HSP90i, in a clinical scenario. In conclusion, this is a very interesting initial study with considerable therapeutic potential.

\section{References}

1. Aceros H, Borie M, Ribeiro RVP, Stevens L-M, Maltais S, Der Sarkissian S, et al. Novel heat shock protein 90 inhibitor improves cardiac recovery in a rodent model of donation after circulatory death. J Thorac Cardiovasc Surg. 2022;163: e187-97.

2. Soti C, Nagy E, Giricz Z, Vigh L, Csermely P, Ferdinandy P. Heat shock proteins as emerging therapeutic targets. Br J Pharmacol. 2005;146:769-80.

3. Aceros H, Der Sarkissian S, Borie M. Stevens LM, Mansour S, Noiseux N. Celastrol-type HSP90 modulators allow for potent cardioprotective effects. Life Sci. 2019;227:8-19.

4. Kearns MJ, Miller SD, Cheung A, Bashir J, Wong S, Seidman MA, et al. A rodent model of cardiac donation after circulatory death and novel biomarkers of cardiac viability during ex vivo heart perfusion. Transplantation. 2017;101:e231-9.

5. Hameed I, Gaudino M. Commentary: do not kill (especially for nothing). J Thorac Cardiovasc Surg. 2019;158:1557-8. 